\title{
Exploring the Potential of ToxCast Data in Supporting Read-Across for Evaluation of Food Chemical Safety
}

\author{
James W. Firman, * Ans Punt, Mark T. D. Cronin, Alan R. Boobis, Martin F. Wilks, Paul A. Hepburn, \\ Anette Thiel, and Karma C. Fussell
}

Cite This: https://dx.doi.org/10.1021/acs.chemrestox.0c00240

Read Online

\section{ACCESS 1}

Џ Metrics \& More

Article Recommendations

Supporting Information

ABSTRACT: The intention of this study was to determine the utility of highthroughput screening (HTS) data, as exemplified by ToxCast and Tox 21 , for application in toxicological read-across in food-relevant chemicals. Key questions were addressed on the extent to which the HTS data could provide information enabling (1) the elucidation of underlying bioactivities associated with apical toxicological outcomes, (2) the closing of existing toxicological data gaps, and (3) the definition of the boundaries of chemical space across which bioactivity could reliably be extrapolated. Results revealed that many biological targets apparently activated within the chemical groupings lack, at this time, validated toxicity pathway associations. Therefore, as means of providing proof-of-principle, a comparatively well-characterized end point-

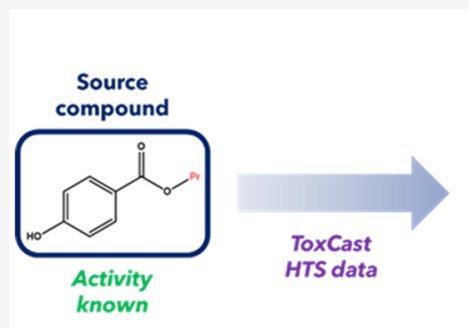
Targets estrogenicity-was selected for evaluation. This was facilitated through the preparation of two exploratory case studies, focusing upon groupings of paraben-gallates and pyranone-type compounds (notably flavonoids). Within both, the HTS data were seen to reflect estrogenic potencies in a manner which broadly corresponded to established structure-activity group relationships, with parabens and flavonoids displaying greater estrogen receptor affinity than benzoate esters and alternative pyranone-containing molecules, respectively. As such, utility in the identification of out-of-domain compounds was demonstrated, indicating potential for application in addressing point (3) as detailed above.

\section{INTRODUCTION}

The traditional use of animals in safety assessment is facing increasing scientific scrutiny as to its appropriateness. Coupled with the associated legal and ethical concerns, this has served to increase urgency in the development of new approaches aimed at determining and predicting adverse effects. ${ }^{1-3}$ In order that chemical regulatory requirements might be met, the generation of novel strategies for human health risk assessment across the range of established and newly marketed compounds has emerged as a necessity.

While resources such as PubChem, ChEMBL, or the OECD QSAR Toolbox provide accessible repositories for existing bioassay outcomes, the efforts of high-throughput screening (HTS) endeavors are further expanding the coverage of compound-biological target interactions. ${ }^{4-6}$ Among the largest and most ambitious HTS enterprises to date is the United States federal collaboration incorporating Tox 21 and ToxCast (referred to jointly henceforth by the descriptor "ToxCast"). Since inception in 2007, these have seen over 9000 chemicals tested across up to 1200 bioassays spanning a variety of in vitro systems. ${ }^{7,8}$ End points screened encompass a broad range of targets, including nuclear receptor interaction and genetic transcription regulation, induction of oxidative stress, enzyme activation, and changes in organelle-specific functionality.
In a recent publication, Punt et al. explored the possibilities of utilizing ToxCast activity data within risk-benefit assessment of food-relevant chemicals. ${ }^{9}$ A collection of more than 500 food substances, drawn from a list specified within Karmaus et al., were examined to obtain insight into their associated biological targets. ${ }^{10,11}$ In doing so, compounds were grouped in accordance with their structural similarity and with their functional uses in food. Activities against ToxCast end points were examined and compared across the chemical groupings, from which patterns of activity could be visualized and inferences as to shared properties drawn.

The exercise of grouping entries according to chemical similarity constitutes a key preliminary stage within the performance of toxicological read-across. ${ }^{12}$ This technique, which derives its methodology from the principle that compounds related in chemical structure (or similar defining characteristic) often display similar patterns of biological activity, has been developed as a means through which gaps

Special Issue: Computational Toxicology

Received: June 12, 2020 
within the data landscape might effectively be filled. ${ }^{13}$ Classical read-across has typically centered upon extrapolation and interpolation of in vivo and in vitro toxicological outcomes, with chemical similarity between compounds then used in a hypothesis-driven fashion in order to propose the sharing of biological effects. ${ }^{14}$ However, the demands of regulatory frameworks to ensure greater confidence in such predictions have spurred efforts to establish enhanced mechanistic grounding in support of the conclusions drawn. ${ }^{15-19}$ This desire has manifested itself in the emergence of the adverse outcome pathway (AOP) paradigm, a framework linking defined molecular-level interaction between compound and biological target or system (molecular initiating event or MIE) with ultimate adverse outcome ( $\mathrm{AO}$ ) through intermediate key events. $^{20,21}$

The insights which ToxCast data may provide concerning mechanisms of toxicity hold great potential for application within read-across, particularly with respect to the avoidance of additional studies within animals. In this context, ToxCast outcomes may assist in (1) the elucidation of underlying bioactivities associated with apical toxicological outcomes, (2) the closing and identification of data gaps (defining an end point for a chemical based on observed biological activity of an analogue), and (3) defining the boundaries of chemical space across which analogues may be inferred to elicit shared biological effects (incorporating structure-activity relationships).

The intention of this study was to build upon the analysis reported by Punt et al., in order to discern whether the associations between chemical structure and in vitro responses that had been identified had potential for adaptation to such read-across purposes. ${ }^{9}$ The basis of this assessment is in the structural groupings established within this earlier publication, alongside shared biological targets as identified through ToxCast outcomes. As such, mapping of ToxCast activities to these chemical groups constituted a fundamental preliminary exercise. Focusing upon estrogenicity for proof of principle, exploratory case studies are presented covering two distinct chemical families: the paraben-gallate grouping and pyranone-type compounds (incorporating flavonoids).

\section{MATERIALS AND METHODS}

2.1. Grouping and Classification of Chemicals with Respect to Structure. Processing and grouping of chemicals proceeded in accordance with the methodology described within Punt et al. ${ }^{9}$ Karmaus et al. identified 563 food additives, which, following removal of mixtures and entries possessing undefined composition, were reduced to a working group consisting of 552 single, discrete molecular entities (listed in their entirety in Supplementary Table 1). ${ }^{10,11}$ These were grouped according either to chemical similarity or to shared functional or physiological role, through a method primarily reliant upon manual judgment, though supplemented with assistance of ChemoTyper software (version 1.0; Molecular Networks, Erlangen, Germany). ${ }^{22}$ A three-tier system of classification was adopted, extending from broadest level "primary" (e.g., carboxylic acid), through intermediate "secondary" (e.g., carboxylic acid, alkyl) to most defined "tertiary" (e.g., carboxylic acid, alkyl, straight chain). In total, 169 tertiary groupings were identified (summarized in Supplementary Table 2). Those groups consisting of two or fewer compounds were excluded for purposes of further analysis, leaving 102 clusters appropriate for read-across consideration (as elaborated in Supplementary Table 3). The extent of similarity between compounds within each group was estimated through derivation of the average Tanimoto coefficient relating possession of shared structural fragments. This was achieved within $\mathrm{R}$ using the ChemmineR tool, with the utilized code accessible at https://git.wur.nl/Punt001/ilsi toxcast. $^{23,24}$

2.2. Classification of ToxCast Outcomes and Quantification of Activity. Input files containing $\mathrm{AC}_{50}$ values (ac50_Matrix_180918.csv), the corresponding Z-scores (zscore-Matrix_180918.csv), flagged results (AllResults_flags_180918.csv) and assay summary information (Assay_Summary_180918.csv) were retrieved from the United States Environmental Protection Agency's (U.S. EPA's) online ToxCast data repository (10.23645/epacomptox. 6062479.v3). These were combined into a unified data set and filtered as outlined below. Such procedures were facilitated through use of R, where the relevant scripts are available for access at https://git. wageningenur.nl/Punt001/ilsi_toxcast_readacross.

End points describing features associated either with assay background effect or with general cytotoxicity were excluded from analysis (based upon the Assay_Summary_180918.csv files). These included all assays for which the "assay function type" was "background control," the "assay_design_type" was "background reporter" or "viability reporter," the "intended_target_family" was "background measurement," and the "biological_process_target" was "cell death," "cell proliferation," or "cytotoxicity". For the consideration of the effect of general cytotoxicity upon the assay outcomes, results with defined $Z$-scores in excess of 3.0 were considered to hold a reasonable likelihood of representing a selective activity at a given molecular target independent of the burst of activities relating to cell death. ${ }^{25}$ For the evaluation of the shared targets activated by the chemical groups, results with $Z$-scores below 3.0 were excluded. Conversely, within the case studies, compounds that expressed activity with $Z$-scores lower than 3.0 toward the selected target were retained in order to provide a more complete indication of the full range of activities (both specific and nonspecific) held by the chemicals within. It should further be noted that this score is to some extent a function of potency, as compounds with high $\mathrm{AC}_{50}$ values tend to have low separation between measured effect and general cytotoxicity. Owing to the high general presence of flagged outcomes across the spread of ToxCast data (those possessing alerts relating the quality of curve-fitting underlying activity calls), such results were by necessity retained for purposes of analysis.

To locate tertiary groupings to which read-across may potentially be applied, analysis was performed to identify those groups containing multiple compounds active at a shared target. Within the ToxCast data, the "technological_target_official_symbol" (e.g., ESR1, HIF1A, NFKB1, etc.) defines the biological target of an assay (many targets possessing multiple corresponding assays). In instances whereby a minimum of two assays corresponding to a biological target were activated by a two or more compounds within a chemical group, an association between the grouping and the target was registered.

2.3. Preparation of Case Studies. For two selected case studies, those of the paraben-gallate grouping and pyranone-type compounds, data (incorporating $\mathrm{AC}_{50}$ values and derived $Z$-scores) were extracted relating to all estrogen receptor (ER)-associated end points. Such chemical groups were selected to form the focus of these studies owing to the strength of their attested associations with respect to estrogenicity, both in vitro and in vivo (as discussed in Section 3.3). ToxCast contains in total $20 \mathrm{ER}$ assays, including those measuring agonism and antagonism at ESR1 $(\mathrm{ER} \alpha)$ and $\mathrm{ESR} 2(\mathrm{ER} \beta)$ receptor subtypes as well as those specific for neither. For each compound present in a group, the activity profile across this suite of assays was determined. Results were split in accordance with $Z$-score. Scores of lower than 3.0 were held to indicate a potential influence by nonspecific factors such as cytotoxicity, whereas scores greater than 3.0 were conversely interpreted as representing unequivocal, targetspecific effects. Distinction was further drawn between outcomes in assays representing agonistic effects and those describing antagonism. The former were defined as those displaying a "positive" assay signal direction, and the latter as those labeled "negative". While attention is drawn to the presence of data flags associated with individual outcomes, flagged results remain integrated within the analysis.

In order to further assess the applicability of hypotheses, structurally related compounds possessing relevant ToxCast data, 
Table 1. Chemical Groupings Listed in Accordance with Quantity of Targets Matched ${ }^{a}$

\begin{tabular}{|c|c|c|c|c|}
\hline group ID & group title & $\begin{array}{c}\text { target } \\
\text { matches }\end{array}$ & key affected target & key target active hits/assays screened (\%) \\
\hline 55 & Ester: Aromatic acid ester: Paraben-gallate & 13 & ESR1IESR2 & $3 / 3(100 \%)$ \\
\hline 11 & Alcohol: Hydroxybenzene: Phenol, aliphatic substituted & 12 & PTGER2 & $9 / 19(47 \%)$ \\
\hline 33 & Ester: Aliphatic alcohol diester/triester: Alkyl alcohol diester/triester & 10 & NR112 & $7 / 52(13 \%)$ \\
\hline 85 & Ketone: Alkenyl: Ionone/irone & 7 & NR1I2 & $11 / 31(35 \%)$ \\
\hline 102 & Vitamins and derivatives & 7 & NR1I2 & $6 / 57(11 \%)$ \\
\hline 24 & Carboxylic acid: Alkyl: Alkyl, straight chain & 6 & SREBF1 & $2 / 10(20 \%)$ \\
\hline 79 & Heterocycles and polycycles: Oxygen heterocycles: Pyranone & 6 & ESR1IESR2 & $4 / 10(40 \%)$ \\
\hline 90 & Metallic salts (organic) & 6 & NR1I2 & $5 / 32(16 \%)$ \\
\hline 93 & Organosulfur: Thiol & 6 & FOSIJUN & $3 / 13(23 \%)$ \\
\hline 1 & Alcohol: Alkenyl: Alkenyl, primary & 5 & NR1I2 & $9 / 45(20 \%)$ \\
\hline 9 & Alcohol: Hydroxybenzene: Alkoxy phenol ether, substituted & 5 & PTGER2 & $3 / 6(50 \%)$ \\
\hline 14 & Alcohol: Phenylalkanol: Phenylalkyl/alkenyl & 5 & MMP9 & $2 / 10(20 \%)$ \\
\hline 15 & Aldehyde: Alkenyl: Alkenyl, acyclic & 5 & NR1I2 & $9 / 30(30 \%)$ \\
\hline 65 & Ester: Lactone: Lactone, five-membered & 5 & NR1I2 & $5 / 53(9 \%)$ \\
\hline 46 & Ester: Alkyl alcohol: Methanol, aliphatic & 4 & RXRB & $2 / 15(15 \%)$ \\
\hline 54 & Ester: Aromatic acid ester: Cinnamate & 4 & NR1I2 & $5 / 22(23 \%)$ \\
\hline 18 & Aldehyde: Aromatic: Benzaldehyde derivatives & 3 & HIF1A & $2 / 24(8 \%)$ \\
\hline 22 & Carboxylic acid: Alkenyl: Alkenyl, straight chain & 3 & PPARG & $2 / 22(9 \%)$ \\
\hline 53 & Ester: Aromatic acid ester: Benzoate & 3 & NR1I2 & $5 / 51(10 \%)$ \\
\hline 62 & Ester: Aromatic alcohol: Phenylethyl alcohol, aliphatic & 3 & TGFB1 & $2 / 8(25 \%)$ \\
\hline 70 & Ether: Aromatic: Aryl methoxy, aliphatic substituted & 3 & NR112 & $3 / 24(12 \%)$ \\
\hline 81 & Hydrocarbon: Terpene & 3 & NR1I2 & $7 / 54(13 \%)$ \\
\hline
\end{tabular}

yet falling outside of the Karmaus et al. data set, were sought. For each chemical within the case study groupings, the "similar compounds" function present on the US EPA CompTox Chemicals Dashboard (https://comptox.epa.gov/dashboard; accessed 2020-09-21) was utilized. ${ }^{26}$ This by default returns all entries present within the database holding a Tanimoto similarity coefficient of $>0.8$ with respect to the parent molecule. A pool consisting of all compounds matching this condition, while simultaneously holding the appropriate data, was created.

\section{RESULTS}

\subsection{Defining Chemical Groups to Which Read-Across} May Potentially Be Applied Based upon ToxCast Outcomes. Activities relating to a total of 244 defined targets (encompassing 600 distinct assays) were mapped across the sum of 102 relevant chemical groupings. In accordance with the criteria described in Section 2.2, a total of 49 chemical groups exhibited activity of at least two substances at a minimum of two assays. A detailed listing describing each relationship may be found in Supplementary Table 4. Appearing in Table 1 is an abridged overview outlining the most prominent groups by quantity of targets affected (presented in full in Supplementary Table 3). In each instance, the key affected target, defined as that possessing a maximal proportion of active hits relative to assays screened, is displayed.

The group consisting of paraben and gallate esters was observed to exhibit the greatest frequency of interaction across ToxCast targets, with activity recorded at 13 sites (estrogenicity being most prominent). Aliphatic-substituted phenols and alkyl alcohol di- and triesters were further notable, registering 12 and 10 associations, respectively. With respect to a key target, NR1I2 (the pregnane X receptor) was represented most commonly.

3.2. Defining Relevant ToxCast Targets for ReadAcross. Of the 244 distinct targets, 46 were found to be
Table 2. Targets Listed by Extent of Activation Across Chemical Groupings

\begin{tabular}{cll}
$\begin{array}{c}\text { groups } \\
\text { matched }\end{array}$ & \multicolumn{1}{c}{ target } & \multicolumn{1}{c}{ protein product } \\
35 & NR1I2 & $\begin{array}{l}\text { pregnane X receptor } \\
\text { estrogen receptor } \alpha\end{array}$ \\
27 & ESR1 & PPAR- $\gamma$ \\
12 & PPARG & Nrf2 \\
11 & NFE2L2 & vitamin D receptor \\
6 & VDR & retinoid X receptor $\alpha$ \\
5 & RXRA & retinoid X receptor $\beta$ \\
5 & RXRB & aryl hydrocarbon receptor \\
4 & AHR & c-Fos \\
3 & FOSIJUN & c-Jun \\
& & prostaglandin receptor E2 \\
3 & PTGER2 & transcription factor 7 \\
3 & TCF7lTCF7L2lLEF1ITCF7L1 & transcription factor 7-like 2 \\
& & lymphoid enhancer-binding \\
& & factor 1 \\
& & transcription factor 7-like 1 \\
& &
\end{tabular}

activated across at least one chemical grouping, with those occurring most frequently outlined in Table 2 (refer to Supplementary Table 5 for complete listing). The pregnane $\mathrm{X}$ receptor (PXR) was identified as most commonly triggered, with activity noted within 35 chemical groups. Owing to the function of this protein in the sensing of xenobiotic substances, it exhibits by necessity a broad specificity with respect to the structure of the molecules which may bind. It is feasible, therefore, that its elevated activation may arise as a product of this physiological role, rather than representing the toxicological response. ${ }^{27}$

Similarly implicated within generalized metabolic and detoxification response is the aryl hydrocarbon receptor (AHR). ${ }^{28} \mathrm{Nrf2}$ (active within 11 groups) is upregulated 
Table 3. Identity, Structure, and Estrogenic Activity Profile for Paraben-Gallate Group as Sourced from (A) Karmaus et al. and (B) Wider ToxCast Repository ${ }^{a}$

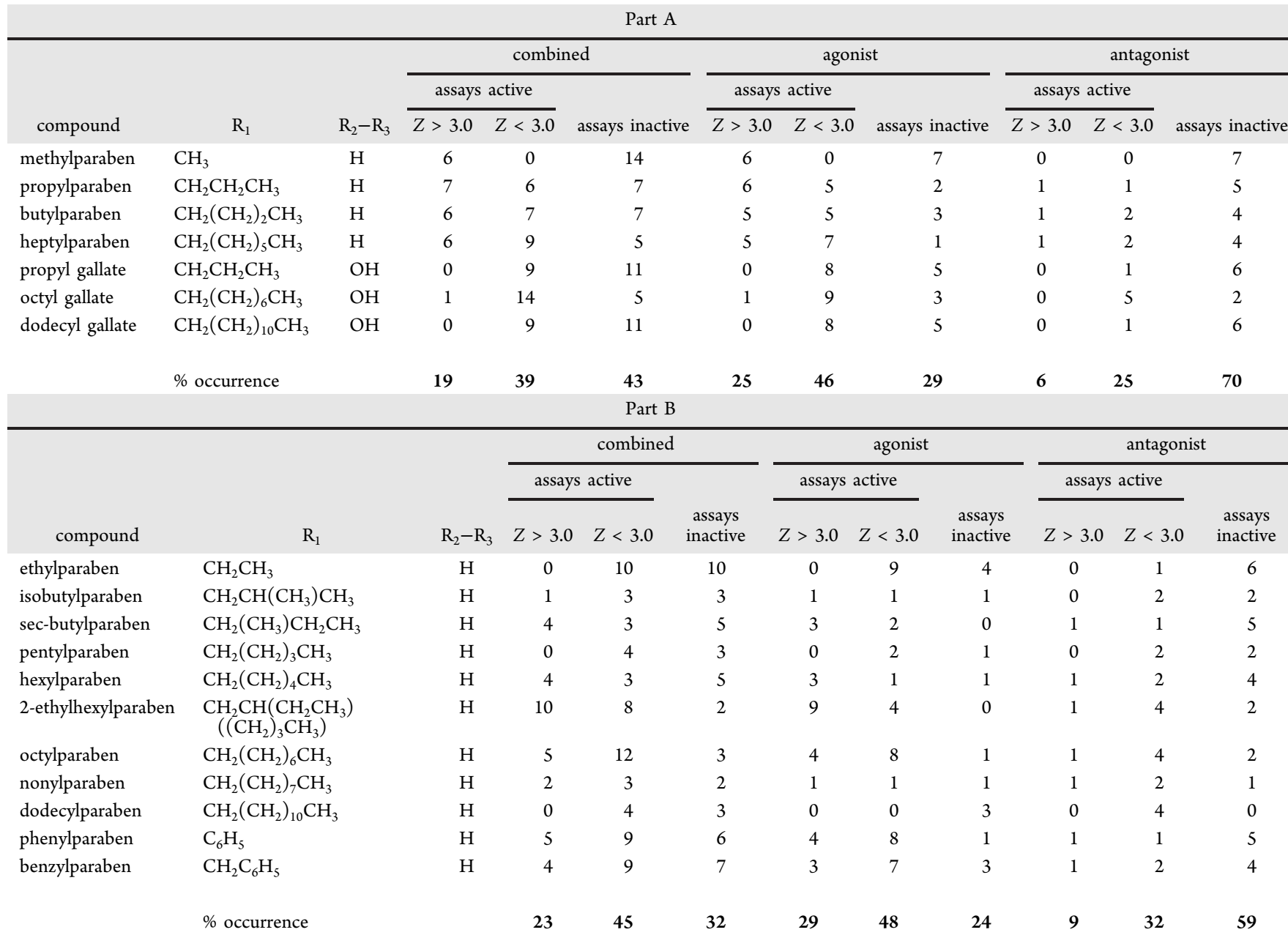

${ }^{a}$ Data relating outcomes at all ER-associated assays (agonist and antagonist) are displayed, grouped in accordance with $Z$-score. Groups $\mathrm{R}_{1}-\mathrm{R}_{3}$ positioned as depicted in Figure 1.

under conditions of oxidative stress and hence may see a greater expression in cells subject to cytotoxicity. ${ }^{29}$ Gene products including PPAR- $\gamma$ and the ESRRA mediate pathways associated with fatty acid metabolism, whereas RXR subtypes are noted as possessing affinity for a varied array of nuclear receptors with which they interact in the formation of heterodimers. ${ }^{30}$ The toxicological impact accompanying the perturbation of these and indeed many of the 46 systems activated through the compound groupings has not been established definitively. Utility toward assisting read-across, both in terms of closing data gaps and elucidating underlying activities, will only become apparent once a wider understanding both of the assay target role within AOPs and of the fundamental limitations arising from the inherent differences present between HTS and alternative systems has progressed.

3.3. Read-Across Case Studies. Taking into account the uncertainty related to the association of various targets with in vivo or clinical manifestation of toxicity, a focus was placed upon defining the translatability of ToxCast outcomes toward end points which serve as the established MIE for definitive, identifiable adverse effects. The estrogen receptor provided the greatest promise in this regard, since there is an extensive array of assays that cover this target and also a clear association with reproductive and developmental toxicity in vivo. ${ }^{31}$ Accordingly, the following studies present assessments of the capacity of the ToxCast data to support read-across (which may be performed either quantitatively or qualitatively) of estrogenicity within two structural groupings, both of which possess corroborating evidence through alternative assay systems (both in vivo and in vitro) attesting the presence of the effect and are furthermore large enough in terms of membership that appropriate conclusions may be drawn.

3.3.1. Paraben-Gallate Grouping. Detailed in Table 3A, this group consists of seven straight-chain alkyl esters ( $n$-alkyl esters), either of 4-hydroxybenzoic acid (termed "parabens") or of 3,4,5-trihydroxybenzoic acid ("gallates"), and the defining base structure of which is depicted in Figure 1. The average ChemmineR-determined structural similarity, expressed in terms of Tanimoto coefficient, stood at 0.72 . All function as preservatives, with parabens employed for their antibacterial and antifungal efficacy and gallates for their antioxidative effect. $^{32,33}$ A total of 13 distinct target end points were activated, as outlined in Table 4.

The estrogenic effect is strongly suggested, with activity evident at both $\mathrm{ER} \alpha$ and $\mathrm{ER} \beta$. A breakdown of compound-bycompound activity profile is displayed in Table $3 \mathrm{~A}$, with differentiation present with respect to nature of receptor interaction (agonism or antagonism) and $Z$-score. In all, 58\% 


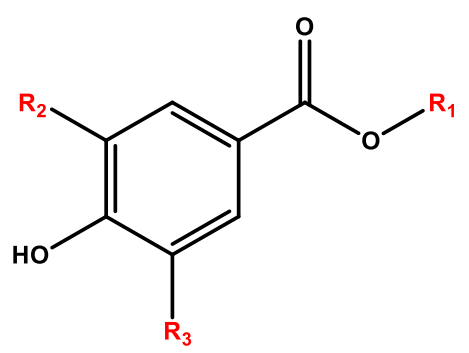

Figure 1. Structural core common to all compounds within the paraben-gallate grouping.

of results across all seven compounds return positive for estrogenicity (19\% when limited to $Z$-scores in excess of 3.0 ). Further resolution revealed that activity at agonism-associated assays was more pronounced than at those indicative of antagonism, with $71 \%$ positivity registered for the latter and $31 \%$ for the former (irrespective of $Z$-score).

Such findings of estrogenicity are supported through the wider literature, with in vitro studies indicating agonistic activity of parabens at each receptor isoform-albeit with potencies substantially reduced relative to those of endogenous estrogens. ${ }^{34-36}$ Outcomes of in vivo studies of parabens again imply an association with estrogen-mediated reproductive dysfunction, confined however to elevated levels of exposure. ${ }^{37,38}$ Gallate esters have yet to be characterized in such detail, yet are recorded as displaying both agonistic and antagonistic activity for these receptors. ${ }^{39-41}$

In order to assess the wider applicability of these associations, a selection of suitably similar compounds holding ToxCast data, yet falling outside of the Karmaus list, was sought. Through use of methodology described in Section 2.3, 11 such chemicals were identified (as listed in Table 3B). Each was a paraben: nine alkyl (in common with those previously considered) and two aryl, with a mean Tanimoto coefficient of 0.73 . Their activity profiles across the suite of estrogenic assays showed close concordance with those within the Karmaus set. In all, $68 \%$ of outcomes were active (23\% with a $Z$-score > 3.0): $77 \%$ among the agonism-related assays and $41 \%$ among antagonist.

Figure 2 provides a graphical representation of activity at individual assay level, spanning the full range of compounds described above. Among $n$-alkyl parabens, a general increase in estrogenic agonist potency was apparent with chain length, moving from methyl to heptyl before falling off from octyl onward. Such an initial increase is corroborated through the outcomes of previous studies, although data on heavier analogues (pentylparaben and above) are lacking. ${ }^{35,38,42}$ More active still than the straight-chain compounds were the branched equivalents: sec-butylparaben and 2-ethylhexylparaben. Enhanced estrogenicity accompanying such branching has been noted in prior studies. ${ }^{43,44}$ Matching these latter analogues in terms of activity were the two arylparabens: phenyl and benzyl. Once again, their greater apparent toxic potential relative to the $n$-alkyl series accords with trends established through alternative, well-recognized assay systems. ${ }^{45}$ Both propyl and octyl gallates displayed a reduced potency relative to the corresponding parabens. Antagonist activity remained uniformly low across all examined compounds, including those gallates for which evidence of such capacity had been reported previously. ${ }^{39-41}$

While constructing read-across groups, it is necessary to define the degree of structural variance across which prediction may reliably be extended (domain of applicability). Both parabens and gallates are considered to display affinity for the ER as a consequence of their 4-OH hydroxyl units, which in turn mimic the identical group present at the A-ring of endogenous estrogens. ${ }^{46}$ Simultaneously, an alkyl substituent at the ester linkage is deemed highly desirable as a means of supporting hydrophobic interactions otherwise facilitated by the greater steroid structure. In order to determine the extent to which removal of either feature influences the receptor affinity relative to parabens and gallates, ToxCast estrogenic activity of a selection of substituted benzoic acids and benzoate (unsubstituted) and salicylate (ortho-hydroxybenzoic acid) esters was analyzed. Each set was composed of the corresponding grouping compiled from Karmaus chemicals (ID 26, 53, and 57, respectively), supplemented as appropriate with further analogues sourced through use of techniques described in Section 2.3. Existing evidence suggests the existence of a very limited estrogenic potential within these classes, falling notably beneath that of parabens upon direct comparison. ${ }^{47,48}$ Appropriately, salicylates, benzoate esters, and substituted benzoic acids (16\%, $9 \%$ and 5\% active, respectively, disregarding $Z$-score distinction) showed a greatly reduced potency across the sum of ER-linked assays. While these results are depicted graphically alongside those of the paraben-gallate grouping in Figure 2, complete descriptions may be found in Supplementary Table 6. A feature of these additional classes, as visible in Figure 2, is the greater prevalence of flags

Table 4. ToxCast Activity Profile for Paraben-Gallate Group, Depicting All Matched Targets

\begin{tabular}{|c|c|c|c|c|}
\hline target & assays screened & assays active & active hit \% & protein product \\
\hline ESR1|ESR2 & 3 & 3 & 100 & estrogen receptors $\alpha, \beta$ \\
\hline PTGER2 & 9 & 4 & 44.4 & prostaglandin receptor E2 \\
\hline ESR2 & 16 & 7 & 43.8 & estrogen receptor $\beta$ \\
\hline TSPO & 6 & 2 & 33.3 & translocator protein \\
\hline CYP1A2 & 10 & 3 & 30 & cytochrome P450 1A2 \\
\hline ESR1 & 56 & 14 & 25 & estrogen receptor $\alpha$ \\
\hline ARIAr & 9 & 2 & 22.2 & androgen receptor \\
\hline NFE2L2 & 17 & 3 & 17.6 & $\mathrm{Nrf2}$ \\
\hline GLI1 & 12 & 2 & 16.7 & zinc finger protein GLI1 \\
\hline ESRRA & 36 & 4 & 11.1 & estrogen-related receptor $\alpha$ \\
\hline NR1I2 & 30 & 3 & 10 & pregnane $\mathrm{X}$ receptor \\
\hline VCAM1 & 47 & 3 & 6.4 & vascular cell adhesion protein 1 \\
\hline TGFB1 & 33 & 2 & 6.1 & transforming growth factor $\beta 1$ \\
\hline
\end{tabular}



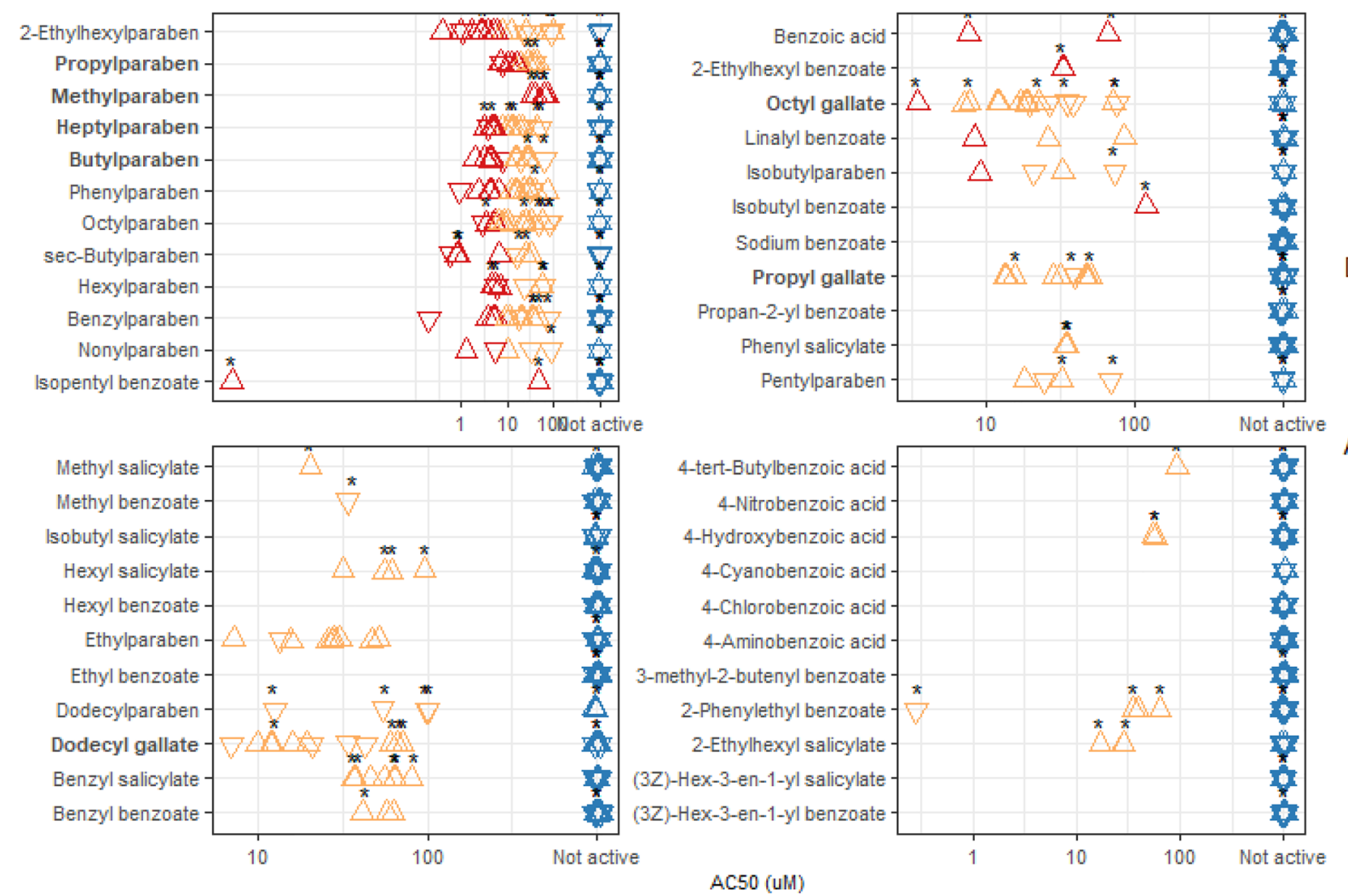

Direction

$\triangle$ gain

$\nabla$ loss

Activity

- ZScore $>3$

- ZScore $<3$

- Not active

Figure 2. Assay-level estrogenic activities of compounds within paraben-gallate and related groups, expressed in terms of micromolar $\mathrm{AC}_{50} . \mathrm{Z}$ scores $>3.0$ are colored red, while those lower are highlighted yellow. Direction "gain" and "loss" represents assays descriptive of agonist and antagonist effects, respectively. Presence of data flags indicated by accompanying asterisk symbol.

accompanying apparently active outcomes. A particularly notable example of this phenomenon may be found in the instance of isopentyl benzoate.

3.3.2. Pyranone. The cluster of pyranone-type compounds present within the Karmaus list consists of eight compoundsthe identities and structures appear in Table 5A. Incorporated are molecules featuring either the 4-pyranone or 2-pyranone moieties (relevant units highlighted red). It is apparent that estrogenic influence again dominates the activity profile of this set, which spans six targets in total (listed in Table 6). A more detailed inspection of the data revealed that this estrogenicity is a feature almost exclusively of the three flavonoid components: daidzein, genistein, and, to a lesser degree, quercetin. Each is, at least to some extent, acknowledged as a phytoestrogen, with evidence attesting to existing both in vitro and in vivo. ${ }^{49,50}$ Relative agonist potencies across individual ER end points followed a general trend, with genistein appearing to have the highest receptor binding affinity, followed by daidzein and then eventually by quercetin (which had $\mathrm{AC}_{50}$ values orders of magnitude greater than the others, as depicted in Figure 3). This mirrors observations recorded within other in vitro systems. ${ }^{51,52}$ As with the grouping of parabens and gallates, all three potencies are greatly reduced relative to those of endogenous estrogens. Antagonist activity was, similarly, consistently low. Quercetin was considered to be borderline positive for $\operatorname{ER} \alpha$ and $\operatorname{ER} \beta$-mediated activities, while maltol and ethyl maltol, whose positive results were isolated and in direct contrast to the negative majority of findings, were judged essentially inactive. This highlights a potential advantage of the approach that weak activity of one analogue may be supported by stronger activity of other structurally related compounds in a group, contributing to a weight-of-evidence assessment.

It is the range variety of biological activities, combined with the diversity of the class of structures responsible (reflected in the comparatively low average Tanimoto similarity score of 0.45), that has the potential to render read-across within this chemical group challenging. Methodology identical to that described in Section 2.3 (and further applied within the paraben-gallate study) was adopted in order to identify a cohort of structurally associated compounds lying outside of the Karmaus list. Fourteen such chemicals were identified (present in Table 5B) with a mean Tanimoto coefficient of 0.5.

Five of this number were coumarin derivatives (including 4methyl-umbelliferone and 5-methoxypsoralen). Appropriately, their apparent estrogenicity-both agonist and antagonistwas minimal. As such, the data can be utilized to draw a greater confidence in reading-across inactivity to all similar compounds. Activity among the alternative classes, both flavone and isoflavone, was however less immediately amenable to simple extrapolation. In contrast to quercetin, which exhibits only moderate potency, the hydroxylated flavones apigenin, kaempferol, and chrysin each appeared as highly active agonists akin to isoflavones genistein and daidzein. Among the nonKarmaus isoflavones were biochanin A, ipriflavone, and formononetin. While the former displays activity in line with its counterparts such as genistein, the latter two were markedly more inert. Such outcomes suggest that patterns of hydroxylation and methoxylation impact upon affinity and that such factors must be considered alongside the nature of the central scaffold when inferring outcomes within a readacross exercise, a point further illustrated by the comparative 


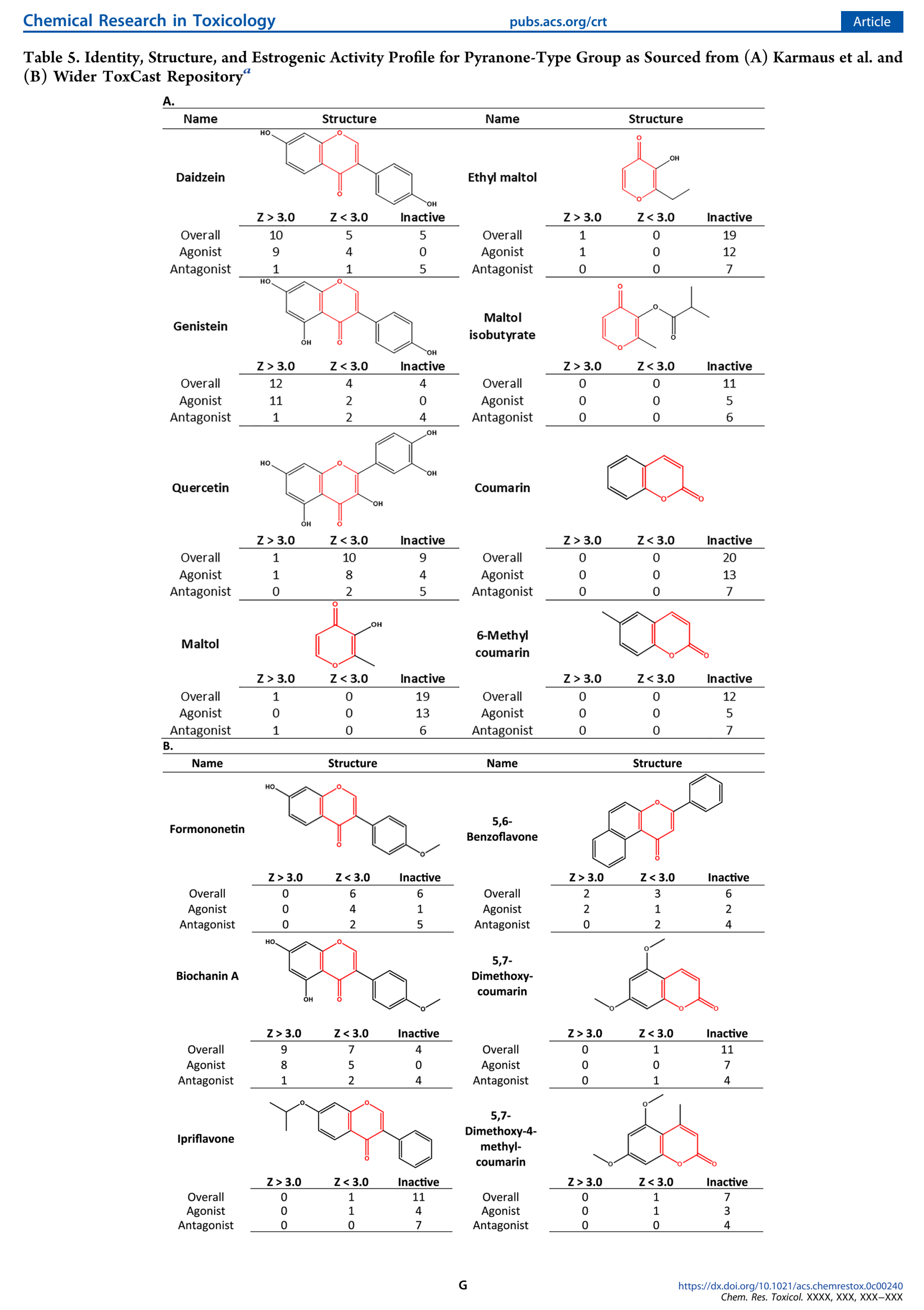


Table 5. continued

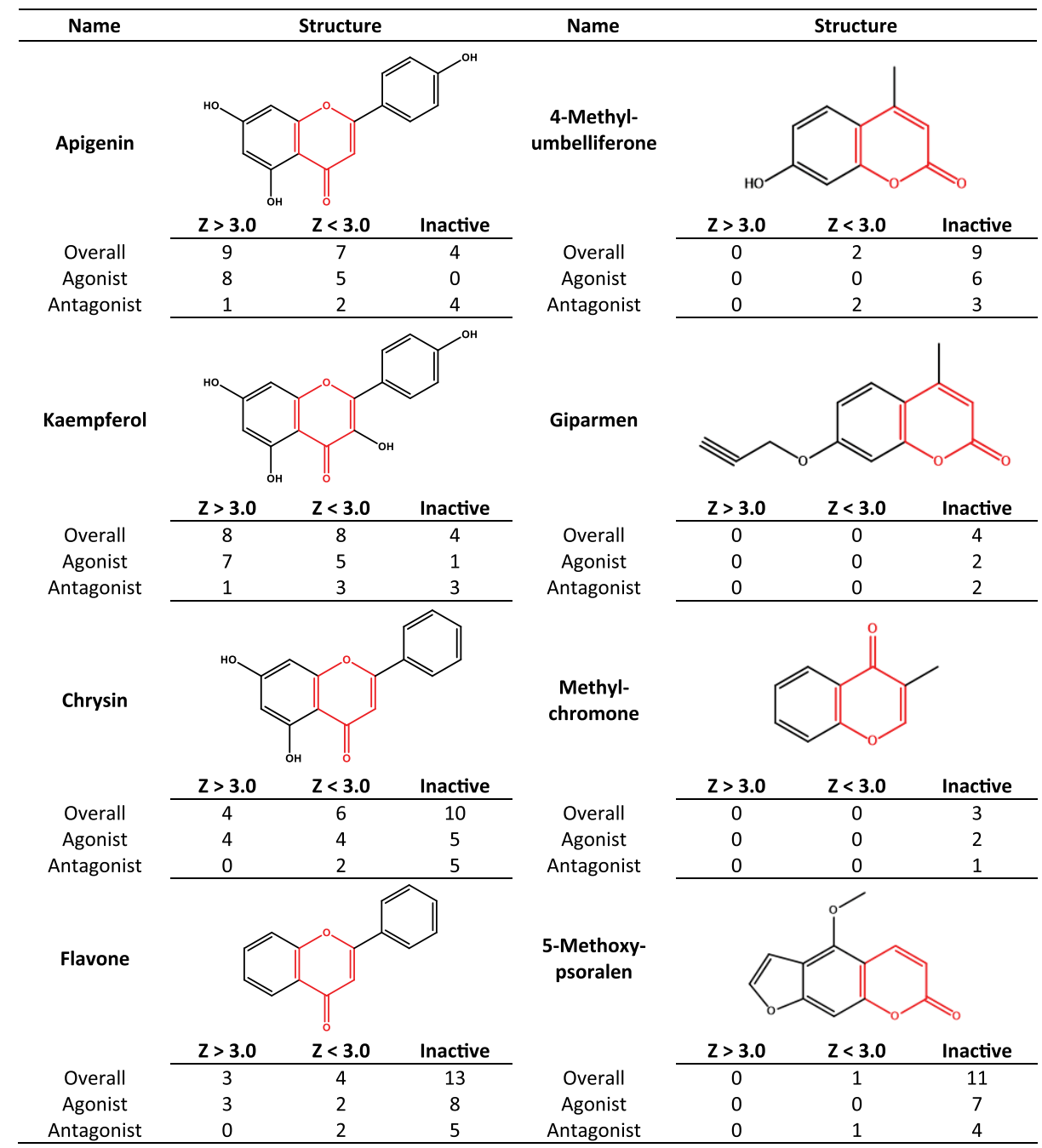

${ }^{a}$ Data relating outcomes at all ER-associated assays (agonist and antagonist) are displayed, classified in accordance with $Z$-score. Pyranone moiety highlighted red.

Table 6. ToxCast Activity Profile for Pyranone-Type Group, Depicting All Matched Targets

\begin{tabular}{lcccl}
\multicolumn{1}{c}{ target } & $\begin{array}{c}\text { assays } \\
\text { screened }\end{array}$ & $\begin{array}{c}\text { assays } \\
\text { active }\end{array}$ & $\begin{array}{c}\text { active } \\
\text { hit } \%\end{array}$ & \multicolumn{1}{c}{ protein product } \\
ESR1IESR2 & 10 & 4 & 40 & estrogen receptors $\alpha, \beta$ \\
ESR2 & 24 & 7 & 29.2 & estrogen receptor $\beta$ \\
ESR1 & 90 & 14 & 16 & estrogen receptor $\alpha$ \\
NFE2L2 & 21 & 3 & 14.3 & Nrf2 \\
F3 & 23 & 2 & 8.7 & tissue factor \\
NR1I3 & 55 & 2 & 3.6 & $\begin{array}{c}\text { constitutive androstane } \\
\text { receptor }\end{array}$ \\
& & & & \\
\hline
\end{tabular}

inactivity of the unsubstituted flavonoids flavone and 5,6benzoflavone. It is appropriate to consider, however, that a variety of factors independent of ligand-receptor SAR (as discussed in Section 3.2) may influence the apparent activity within these systems.

\section{DISCUSSION}

Traditional read-across has focused upon established end points within toxicology for which data have been most widely available, of particular prominence is the no observed effect level (NOEL) quantities obtained following acute or repeated- dose administration of compounds within test animal species. Such an exercise typically relies upon chemical similarity in the framing of boundaries across which predictions (generally limited to organ or whole-organism level outcomes) may be extended. Aside from the associated ethical considerations, such protocols are costly and time-consuming and may lack both translational relevance and mechanistic grounding. In vitro HTS data, exemplified by ToxCast and Tox21, offer potential solutions to a number of these issues, enabling generation of volumes of pathway-specific data without recourse to in vivo methodology. A future vision for a more mechanistically anchored read-across may include incorporation of such findings, and as such, their utility and shortcomings must be assessed. Within this study, key questions were raised as to the extent to which the HTS data could provide assistance enabling (1) the elucidation of underlying bioactivities associated with apical toxicological outcomes, (2) the closing of existing toxicological data gaps, and (3) the definition of the boundaries of chemical space across which bioactivity could reliably be extrapolated.

In order to appreciate the challenges associated with addressing these points, it is first necessary to consider the distribution and general interpretability of the biological 

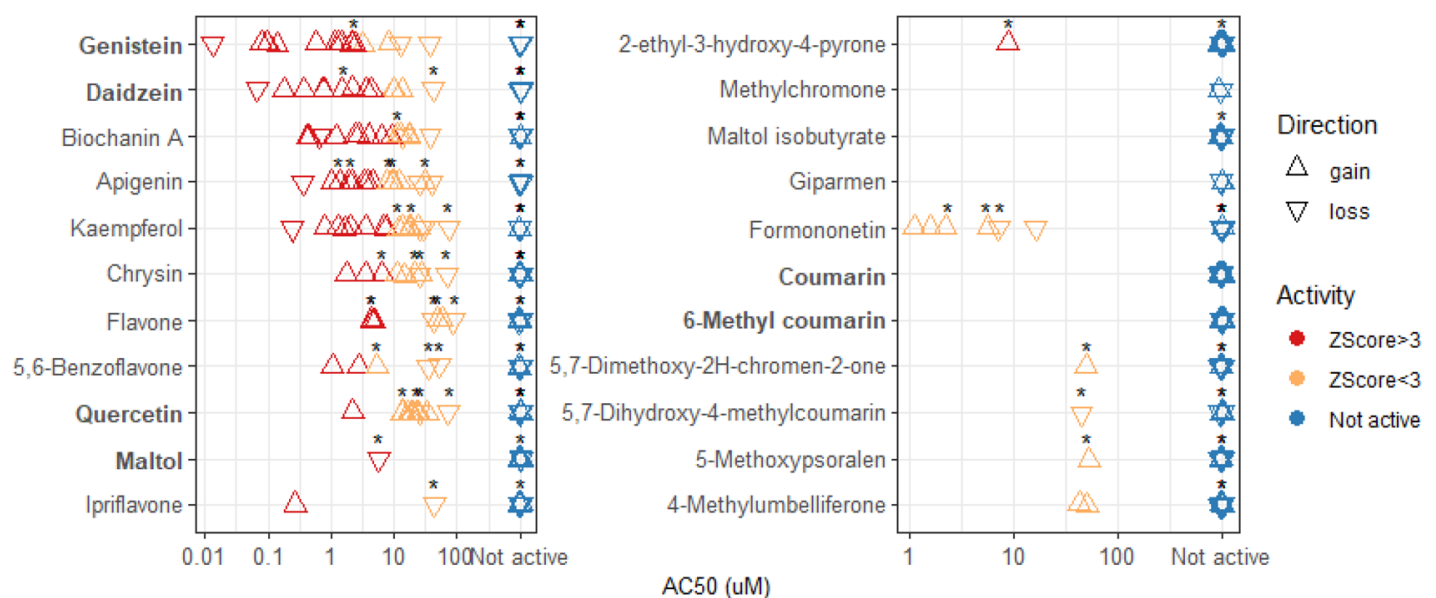

Figure 3. Assay-level estrogenic activities of compounds within the pyranone-type group, expressed in terms of micromolar $\mathrm{AC}_{50}$. Z-scores $>3.0$ are colored red, while those lower are highlighted yellow. Direction "gain" and "loss" represent assays descriptive of agonist and antagonist effect, respectively. Presence of data flags indicated by accompanying asterisk symbol.

associations suggested. Of the 102 suitably defined chemical groups, 49 displayed activity against at least one target. Across all, a total of 165 putative relationships were recorded. It should be noted that of the 244 targets identified, approximately four-fifths were inactive across all groups. Among the most commonly triggered assays were those relating to the general sensing of, and response to, xenobiotic presence, including transcriptional regulators PXR (activated across 35 groups) and AHR (four groups). ${ }^{53}$ Owing to the necessary broadness of their substrate specificity, widespread stimulation under conditions utilized within the ToxCast assays is to be anticipated and may be considered an adaptive physiological response facilitating metabolism and detoxification. PPAR $\gamma$ similarly possesses a large, adaptive binding pocket capable of facilitating activity of a wide selection of ligands - a factor which is correspondingly likely to account for the variety of groups (12 in total) which share activity against it. $^{54}$ Induction of Nrf2, a transcription factor associated with mediation of defense toward oxidative stress, was noted within 11 chemical groups. It is plausible that the extent of its enhancement within the HTS protocols arises as a function of general stress placed upon cells by elevated xenobiotic concentration. ${ }^{29}$ RXR subtypes $\alpha$ and $\beta$ are in combination triggered across 10 groups. Ready interpretation of the relevance of these findings is confounded by the promiscuity of such proteins as dimerization partners for PXR and CAR, together with a variety of nuclear receptors including PPAR, RAR, LXR, FXR, and TR. ${ }^{30}$

With doubt cast over the relevance and translatability of the aforementioned end points with respect to definable adverse outcome, it was necessary to probe associations within the variety of less commonly triggered targets. In total, 38 of the 46 matched targets were present within three or fewer groups. In a number of instances, the functions of such entities have been characterized: HIF1A, p53, and VEGF1 (FLT1) have, for example, each been studied extensively. Others, however, remain more obscure. It is likely that many of the associations between the ToxCast end point and in vivo effect (the subject of the first read-across applicability question raised within this study) will remain unknown until knowledge of the underlying biological pathways has expanded. This will require advancement in the general understanding of the influences of gene products upon the progression of pathology, an endeavor requiring progression across "omics" fields. ${ }^{55}$ Publicly accessible resources including Reactome and KEGG are among a number of established repositories holding information concerning the connections underlying physiological systems. ${ }^{56,57}$ Similarly, the Comparative Toxicogenomics Database explicitly seeks to associate the disturbance of gene expression with defined toxicological end points. ${ }^{58}$ Only through a continuation of research into these interactions, and subsequent integration into informed AOPs, will rationalization of the wealth of data available be realized. ${ }^{21}$ It should be noted that the addressing of second question posed-that of whether the recovered data could be adapted for the closing of existing toxicological data gaps-is very much dependent upon the establishment of such mechanistic links. Accordingly, it is apparent that the utility of the examined HTS outcomes for this purpose at present remains limited.

As such, the focus was limited to a pair of comparatively well-characterized relationships: the xenoestrogenicity of both the parabens and of pyranone-type compounds (notably flavonoids). ${ }^{34,59}$ ToxCast contains a variety of assays characterizing agonist and antagonist effects at both ER subtypes, enabling an apparently reliable characterization of compound action at these molecular targets. Indeed, many of the most promising studies to date examining the predictive potential of the ToxCast data have focused upon endocrine-mediated end points through not only the estrogenic but also the androgenic receptors. ${ }^{6-64}$ It was noted that not only did these assays reflect the general presence of estrogenicity within the examined groups, but that the potency of the suggested effects in some instances correlated with structure-activity trends indicated through other assay systems. Resolution to such a level is of course highly desirable within practical read-across scenarios, where subtle structural alterations across a grouping of closely related compounds may be associated with variations in activity. Within the paraben-gallate grouping, it was observed that aryl and branched-chain alkyl parabens showed a greater agonist affinity for the receptors than did the straightchain equivalents. Among these $n$-alkyl analogues, a trend of increasing agonistic activity was seen from methylparaben to butylparaben, with each more potent than its counterpart gallate. Evidence of ER antagonism was present only to a minimal degree in all. Within the pyranone group, however, concordance with accepted SAR among flavonoids and 
isoflavonoids was not so readily apparent. While it is acknowledged that isoflavonoids generally display greater estrogenic potency than do the corresponding flavones, this trend was not universally mirrored in ToxCast outcomes-a factor likely attributable in part to the variance in hydroxyl and methoxy substitution patterns additionally present. ${ }^{65,66}$

Within both case studies, a clear contrast was observed between the estrogenic profiles of those compounds with widely attested estrogenic potential and those structural relatives without. For example, marked differences were noted in the activity of parabens and gallates relative to benzoate esters and salicylates (lacking the essential 4-OH binding group) and also to the substituted benzoic acids (without the alkyl ester substituent). Hydroxylated isoflavonoids and flavonoids were substantially more potent than their equivalents not possessive of appropriate hydrogen-bonding units (such as flavone and 5,6-benzoflavone). Such results suggest that ToxCast forms a relevant source of information primarily in defining the boundaries of the chemical space across which analogues may elicit certain biological effects. In doing so, this answers effectively the third read-across question ventured at the commencement of the study. Adaptation of this capacity may facilitate the development of tools enhancing confidence in the suitability of compounds for inclusion within putative read-across categories, offering further dimensionality when integrated alongside those methods solely considerate of chemical structure such as molecular fingerprints, structural alerts, and physicochemical property descriptors. ${ }^{12}$

It should be recalled that relating the outcomes of in vitro assays to defined in vivo effects is an inherently challenging exercise, with a general limitation of the most current HTS approaches such as ToxCast being the inability of the constituent assays to account for xenobiotic bioactivation and metabolism, factors integral in determining not only the fate of but in some cases the bioactivity of administered compounds within living organisms. ${ }^{67}$ Additional issues include the presence of gaps, often substantial, within the data landscape (which undoubtedly contributed to the lack of matches relating to the majority of targets present) and further technical challenges associated with the processing of chemicals varying intrinsically with regards to their volatility, stability, and proclivity to bind to materials. ${ }^{68}$ Previous studies conducted with the intention of adapting ToxCast end points for the construction of predictive toxicological models have noted varying degrees of performance, dependent upon the systems examined. ${ }^{69}$ Associations between nuclear receptor assay activation (notably of the PPAR family) and hepatotoxicity have additionally been observed, while further promising outcomes were noted in efforts to detect signatures representing impairment to vascular development. ${ }^{70,71}$ However, attempts to discern relationships with neurotoxicity and adipogenesis, among others, have in contrast proved less successful. $^{60,72,73}$

In conclusion, this study has enabled a preliminary exploration of the utility of HTS data, as exemplified by results from ToxCast, for application within chemical readacross for food relevant chemicals. Uncertainties with regards to the relevance of many of the target hits toward apical outcome limit application at the current time, although it is likely that with an appreciation of their place within AOPs, the applicability of the findings will likewise expand. Representative case studies indicate a promising ability to replicate estrogenic effects found within the paraben-gallate grouping and also within pyranone-containing flavonoid compounds, discriminating out-of-domain structural relatives and mirroring, to an extent, in-group SAR.

\section{ASSOCIATED CONTENT}

\section{Supporting Information}

The Supporting Information is available free of charge at https://pubs.acs.org/doi/10.1021/acs.chemrestox.0c00240.

Supplementary Table 1: Identity of compounds present within Karmaus et al. list, accompanied by their primary, secondary, and tertiary structural grouping assignments. Supplementary Table 2: Overview of compiled chemical groupings. Supplementary Table 3: Chemical groupings eligible for read-across consideration. Supplementary Table 4: Unabridged listing of chemical grouping-target associations. Supplementary Table 5: ToxCast targets ordered by extent of activation across chemical groupings. Supplementary Table 6: ToxCast estrogenic activity profiles for compound clusters considered alongside paraben-gallates (XLSX)

\section{AUTHOR INFORMATION}

\section{Corresponding Author}

James W. Firman - School of Pharmacy and Biomolecular Sciences, Liverpool John Moores University, Liverpool L3 3AF, United Kingdom; 이이.org/0000-0003-0319-1407; Email: J.W.Firman@ljmu.ac.uk

\section{Authors}

Ans Punt - Wageningen Food Safety Research, 6700 AE Wageningen, The Netherlands

Mark T. D. Cronin - School of Pharmacy and Biomolecular Sciences, Liverpool John Moores University, Liverpool L3 3AF, United Kingdom; () orcid.org/0000-0002-6207-4158

Alan R. Boobis - National Heart and Lung Institute, Imperial College London, London W12 ONN, United Kingdom

Martin F. Wilks - Swiss Centre for Applied Human Toxicology and Department of Pharmaceutical Sciences, University of Basel, CH-4055 Basel, Switzerland

Paul A. Hepburn - Safety and Environmental Assurance Centre, Unilever, Sharnbrook MK44 1LQ, United Kingdom

Anette Thiel - DSM Nutritional Products, 4303 Kaiseraugst, Switzerland

Karma C. Fussell - Nestlé Research, CH-1000 Lausanne 26, Switzerland

Complete contact information is available at:

https://pubs.acs.org/10.1021/acs.chemrestox.0c00240

\section{Funding}

Experts are not paid for the time spent on this work; however, the non-industry members within the expert group were offered support for travel and accommodation costs from the New Approaches to Chemical Risk Assessment for Food and Food Ingredients Task Force to attend meetings to discuss the manuscript and a small compensatory sum (honorarium) with the option to decline.

\section{Notes}

The opinions expressed herein and the conclusions of this publication are those of the authors and do not necessarily represent the views of ILSI Europe nor those of its member companies, nor any regulatory authority.

The authors declare no competing financial interest. 


\section{ACKNOWLEDGMENTS}

This work was conducted by an expert group of the European branch of the International Life Sciences Institute, ILSI Europe. The research question addressed in this publication and potential contributing experts in the field were identified by the New Approaches to Chemical Risk Assessment for Food and Food Ingredients Task Force. Industry members of this task force are listed on the ILSI Europe website at https:// ilsi.eu/new-approaches-in-chemical-risk-assessment tfonepager_feb2019/. According to ILSI Europe policies, the EG is composed by at least $50 \%$ of external non-industry members. Once the expert group was formed, the research project was handed over to them to independently refine the research question. Consequently the expert group carried out the work, that is, collecting/analyzing data/information and writing the scientific paper independently of other activities of the task force. The research reported is the result of a scientific evaluation in line with ILSI Europe's framework to provide a precompetitive setting for public-private partnership. ILSI Europe (Dr. Despoina Angeliki Stavropoulou and former ILSI Europe staff members Dr. Bettina Schelkle and Dr. Kirsi Forsberg) facilitated scientific meetings and coordinated the overall project management and administrative tasks relating to the completion of this work. For further information about ILSI Europe, please email info@ilsieurope.be or call +3227710014 .

\section{REFERENCES}

(1) Krewski, D., Acosta, D., Andersen, M., Anderson, H., Bailar, J. C., Boekelheide, K., Brent, R., Charnley, G., Cheung, V. G., Green, S., Kelsey, K. T., Kerkvliet, N. I., Li, A. A., McCray, L., Meyer, O., Patterson, R. D., Pennie, W., Scala, R. A., Solomon, G. M., Stephens, M., Yager, J., and Zeise, L. (2010) Toxicity testing in the 21st century: a vision and a strategy. J. Toxicol. Environ. Health, Part B 13, 51-138.

(2) Gocht, T., Berggren, E., Ahr, H. J., Cotgreave, I., Cronin, M. T. D., Daston, G., Hardy, B., Heinzle, E., Hescheler, J., Knight, D. J., Mahony, C., Peschanski, M., Schwarz, M., Thomas, R. S., Verfaillie, C., White, A., and Whelan, M. (2015) The SEURAT-1 Approach towards Animal Free Human Safety Assessment. Altex-Alternatives to Animal Experimentation 32, 9-24.

(3) Tralau, T., Oelgeschlager, M., Gurtler, R., Heinemeyer, G., Herzler, M., Hofer, T., Itter, H., Kuhl, T., Lange, N., Lorenz, N., Muller-Graf, C., Pabel, U., Pirow, R., Ritz, V., Schafft, H., Schneider, H., Schulz, T., Schumacher, D., Zellmer, S., Fleur-Bol, G., Greiner, M., Lahrssen-Wiederholt, M., Lampen, A., Luch, A., Schonfelder, G., Solecki, R., Wittkowski, R., and Hensel, A. (2015) Regulatory toxicology in the twenty-first century: challenges, perspectives and possible solutions. Arch. Toxicol. 89, 823-850.

(4) Kim, S., Thiessen, P. A., Bolton, E. E., Chen, J., Fu, G., Gindulyte, A., Han, L. Y., He, J. E., He, S. Q., Shoemaker, B. A., Wang, J. Y., Yu, B., Zhang, J., and Bryant, S. H. (2016) PubChem Substance and Compound databases. Nucleic Acids Res. 44, D1202-D1213.

(5) Gaulton, A., Hersey, A., Nowotka, M., Bento, A. P., Chambers, J., Mendez, D., Mutowo, P., Atkinson, F., Bellis, L. J., Cibrian-Uhalte, E., Davies, M., Dedman, N., Karlsson, A., Magarinos, M. P., Overington, J. P., Papadatos, G., Smit, I., and Leach, A. R. (2017) The ChEMBL database in 2017. Nucleic Acids Res. 45, D945-D954.

(6) Dimitrov, S. D., Diderich, R., Sobanski, T., Pavlov, T. S., Chankov, G. V., Chapkanov, A. S., Karakolev, Y. H., Temelkov, S. G., Vasilev, R. A., Gerova, K. D., Kuseva, C. D., Todorova, N. D., Mehmed, A. M., Rasenberg, M., and Mekenyan, O. G. (2016) QSAR Toolbox - workflow and major functionalities. Sar and Qsar in Environmental Research 27, 203-219.

(7) Richard, A. M., Judson, R. S., Houck, K. A., Grulke, C. M., Volarath, P., Thillainadarajah, I., Yang, C. H., Rathman, J., Martin, M. T., Wambaugh, J. F., Knudsen, T. B., Kancherla, J., Mansouri, K.,
Patlewicz, G., Williams, A. J., Little, S. B., Crofton, K. M., and Thomas, R. S. (2016) ToxCast Chemical Landscape: Paving the Road to 21st Century Toxicology. Chem. Res. Toxicol. 29, 1225-1251.

(8) Tice, R. R., Austin, C. P., Kavlock, R. J., and Bucher, J. R. (2013) Improving the Human Hazard Characterization of Chemicals: A Tox21 Update. Environ. Health Perspect. 121, 756-765.

(9) Punt, A., Firman, J., Boobis, A., Cronin, M., Gosling, J. P., Wilks, M. F., Hepburn, P. A., Thiel, A., and Fussell, K. C. (2020) Potential of ToxCast Data in the Safety Assessment of Food Chemicals. Toxicol. Sci. 174, 326-340.

(10) Karmaus, A. L., Filer, D. L., Martin, M. T., and Houck, K. A. (2016) Evaluation of food-relevant chemicals in the ToxCast highthroughput screening program. Food Chem. Toxicol. 92, 188-196.

(11) Karmaus, A. L., Trautman, T. D., Krishan, M., Filer, D. L., and Fix, L. A. (2017) Curation of food-relevant chemicals in ToxCast. Food Chem. Toxicol. 103, 174-182.

(12) Cronin, M. T. D., Madden, J. C., Enoch, S. J., and Roberts, D. (2013) Chemical Toxicity Prediction: Category Formation and ReadAcross, Royal Society of Chemistry, Cambridge, UK.

(13) Patlewicz, G., Ball, N., Becker, R. A., Booth, E. D., Cronin, M. T., Kroese, D., Steup, D., van Ravenzwaay, B., and Hartung, T. (2014) Read-across approaches-misconceptions, promises and challenges ahead. Altex 31, 387-396.

(14) van Leeuwen, K., Schultz, T. W., Henry, T., Diderich, B., and Veith, G. D. (2009) Using chemical categories to fill data gaps in hazard assessment. Sar and Qsar in Environmental Research 20, 207220.

(15) Ball, N., Cronin, M. T., Shen, J., Blackburn, K., Booth, E. D., Bouhifd, M., Donley, E., Egnash, L., Hastings, C., Juberg, D. R., Kleensang, A., Kleinstreuer, N., Kroese, E. D., Lee, A. C., Luechtefeld, T., Maertens, A., Marty, S., Naciff, J. M., Palmer, J., Pamies, D., Penman, M., Richarz, A. N., Russo, D. P., Stuard, S. B., Patlewicz, G., van Ravenzwaay, B., Wu, S., Zhu, H., and Hartung, T. $(2016)$ Toward Good Read-Across Practice (GRAP) guidance. Altex 33, 149-166.

(16) Patlewicz, G., Cronin, M. T. D., Helman, G., Lambert, J. C., Lizarraga, L. E., and Shah, I. (2018) Navigating through the minefield of read-across frameworks: A commentary perspective. Computational Toxicology 6, 39-54.

(17) Przybylak, K. R., Schultz, T. W., Richarz, A.-N., Mellor, C. L., Escher, S. E., and Cronin, M. T. D. (2017) Read-across of 90-day rat oral repeated-dose toxicity: A case study for selected $\beta$-olefinic alcohols. Computational Toxicology 1, 22-32.

(18) Schultz, T. W., Przybylak, K. R., Richarz, A.-N., Mellor, C. L. Escher, S. E., Bradbury, S. P., and Cronin, M. T. D. (2017) Readacross of 90-day rat oral repeated-dose toxicity: A case study for selected n-alkanols. Computational Toxicology 2, 12-19.

(19) Firman, J. W., Patel, A., Date, M., Cronin, M. T. D., and Schultz, T. W. (2018) Read-across of 90-day rodent repeated-dose toxicity: A case study for selected simple aryl alcohol alkyl carboxylic acid esters. Computational Toxicology 7, 1-8.

(20) Ankley, G. T., Bennett, R. S., Erickson, R. J., Hoff, D. J., Hornung, M. W., Johnson, R. D., Mount, D. R., Nichols, J. W., Russom, C. L., Schmieder, P. K., Serrrano, J. A., Tietge, J. E., and Villeneuve, D. L. (2010) Adverse outcome pathways: A conceptual framework to support ecotoxicology research and risk assessment. Environ. Toxicol. Chem. 29, 730-741.

(21) Vinken, M. (2013) The adverse outcome pathway concept: A pragmatic tool in toxicology. Toxicology 312, 158-165.

(22) Yang, C., Tarkhov, A., Marusczyk, J., Bienfait, B., Gasteiger, J., Kleinoeder, T., Magdziarz, T., Sacher, O., Schwab, C. H., Schwoebel, J., Terfloth, L., Arvidson, K., Richard, A., Worth, A., and Rathman, J. (2015) New Publicly Available Chemical Query Language, CSRML, To Support Chemotype Representations for Application to Data Mining and Modeling. J. Chem. Inf. Model. 55, 510-528.

(23) Cao, Y., Charisi, A., Cheng, L.-C., Jiang, T., and Girke, T. (2008) ChemmineR: a compound mining framework for $\mathrm{R}$. Bioinformatics 24, 1733-1734.

(24) (2012) R: A language and environment for statistical computing, $R$ Foundation for Statistical Computing, Vienna, Austria. 
(25) Judson, R., Houck, K., Martin, M., Richard, A. M., Knudsen, T. B., Shah, I., Little, S., Wambaugh, J., Woodrow Setzer, R., Kothiya, P., Phuong, J., Filer, D., Smith, D., Reif, D., Rotroff, D., Kleinstreuer, N., Sipes, N., Xia, M., Huang, R., Crofton, K., and Thomas, R. S. (2016) Editor's Highlight: Analysis of the Effects of Cell Stress and Cytotoxicity on In Vitro Assay Activity Across a Diverse Chemical and Assay Space. Toxicol. Sci. 152, 323-339.

(26) Williams, A. J., Grulke, C. M., Edwards, J., McEachran, A. D., Mansouri, K., Baker, N. C., Patlewicz, G., Shah, I., Wambaugh, J. F., Judson, R. S., and Richard, A. M. (2017) The CompTox Chemistry Dashboard: a community data resource for environmental chemistry. J. Cheminf. 9, 61.

(27) Kliewer, S. A., Goodwin, B., and Willson, T. M. (2002) The nuclear pregnane $\mathrm{X}$ receptor: $\mathrm{A}$ key regulator of xenobiotic metabolism. Endocr. Rev. 23, 687-702.

(28) Kawajiri, K., and Fujii-Kuriyama, Y. (2017) The aryl hydrocarbon receptor: a multifunctional chemical sensor for host defense and homeostatic maintenance. Exp. Anim. 66, 75-89.

(29) Kensler, T. W., Wakabayashi, N., and Biswal, S. (2007) Cell survival responses to environmental stresses via the Keap1-Nrf2-ARE pathway. Annu. Rev. Pharmacol. Toxicol. 47, 89-116.

(30) Dawson, M. I., and Xia, Z. (2012) The retinoid X receptors and their ligands. Biochim. Biophys. Acta, Mol. Cell Biol. Lipids 1821, 2156.

(31) Shanle, E. K., and Xu, W. (2011) Endocrine Disrupting Chemicals Targeting Estrogen Receptor Signaling: Identification and Mechanisms of Action. Chem. Res. Toxicol. 24, 6-19.

(32) Crovetto, S. I., Moreno, E., Dib, A. L., Espigares, M., and Espigares, E. (2017) Bacterial toxicity testing and antibacterial activity of parabens. Toxicol. Environ. Chem. 99, 858-868.

(33) González, M. J., Medina, I., Maldonado, O. S., Lucas, R., and Morales, J. C. (2015) Antioxidant activity of alkyl gallates and glycosyl alkyl gallates in fish oil in water emulsions: relevance of their surface active properties and of the type of emulsifier. Food Chem. 183, 190196.

(34) Bledzka, D., Gromadzinska, J., and Wasowicz, W. (2014) Parabens. From environmental studies to human health. Environ. Int. 67, 27-42.

(35) Byford, J. R., Shaw, L. E., Drew, M. G. B., Pope, G. S., Sauer, M. J., and Darbre, P. D. (2002) Oestrogenic activity of parabens in MCF7 human breast cancer cells. J. Steroid Biochem. Mol. Biol. 80, 49-60.

(36) Watanabe, Y., Kojima, H., Takeuchi, S., Uramaru, N., Ohta, S., and Kitamura, S. (2013) Comparative study on transcriptional activity of 17 parabens mediated by estrogen receptor $\alpha$ and $\beta$ and androgen receptor. Food Chem. Toxicol. 57, 227-234.

(37) Vo, T. T. B., Yoo, Y.-M., Choi, K.-C., and Jeung, E.-B. (2010) Potential estrogenic effect(s) of parabens at the prepubertal stage of a postnatal female rat model. Reprod. Toxicol. 29, 306-316.

(38) Lemini, C., Jaimez, R., Avila, M. E., Franco, Y., Larrea, F., and Lemus, A. E. (2003) In vivo and in vitro estrogen bioactivities of alkyl parabens. Toxicol. Ind. Health 19, 69-79.

(39) ter Veld, M. G. R., Schouten, B., Louisse, J., van Es, D. S., van der Saag, P. T., Rietjens, I., and Murk, A. J. (2006) Estrogenic potency of food-packaging-associated plasticizers and antioxidants as detected in ER alpha and ER beta reporter gene cell lines. J. Agric. Food Chem. 54, 4407-4416.

(40) Amadasi, A., Mozzarelli, A., Meda, C., Maggi, A., and Cozzini, P. (2009) Identification of Xenoestrogens in Food Additives by an Integrated in Silico and in Vitro Approach. Chem. Res. Toxicol. 22, $52-63$.

(41) Pop, A., Berce, C., Bolfa, P., Nagy, A., Catoi, C., Dumitrescu, I. B., Silaghi-Dumitrescu, L., and Loghin, F. (2013) Evaluation of the possible endocrine disruptive effect of butylated hydroxyanisole, butylated hydroxytoluene and propyl gallate in immature female rats. Farmacia 61, 202-211.

(42) Routledge, E. J., Parker, J., Odum, J., Ashby, J., and Sumpter, J. P. (1998) Some alkyl hydroxy benzoate preservatives (parabens) are estrogenic. Toxicol. Appl. Pharmacol. 153, 12-19.
(43) Okubo, T., Yokoyama, Y., Kano, K., and Kano, I. (2001) ERdependent estrogenic activity of parabens assessed by proliferation of human breast cancer MCF-7 cells and expression of ER $\alpha$ and PR. Food Chem. Toxicol. 39, 1225-1232.

(44) Darbre, P. D., Byford, J. R., Shaw, L. E., Horton, R. A., Pope, G. S., and Sauer, M. J. (2002) Oestrogenic activity of isobutylparaben in vitro and in vivo. J. Appl. Toxicol. 22, 219-226.

(45) Golden, R., Gandy, J., and Vollmer, G. (2005) A Review of the Endocrine Activity of Parabens and Implications for Potential Risks to Human Health. Crit. Rev. Toxicol. 35, 435-458.

(46) Lee, S., and Barron, M. G. (2017) Structure-Based Understanding of Binding Affinity and Mode of Estrogen Receptor $\alpha$ Agonists and Antagonists. PLoS One 12, e0169607-e0169607.

(47) Miller, D., Wheals, B. B., Beresford, N., and Sumpter, J. P. (2001) Estrogenic Activity of Phenolic Additives Determined by an In Vitro Yeast Bioassay. Environ. Health Perspect. 109, 133-138.

(48) Morohoshi, K., Yamamoto, H., Kamata, R., Shiraishi, F., Koda, T., and Morita, M. (2005) Estrogenic activity of 37 components of commercial sunscreen lotions evaluated by in vitro assays. Toxicol. In Vitro 19, 457-469.

(49) Cassidy, A., Hanley, B., and Lamuela-Raventos, R. M. (2000) Isoflavones, lignans and stilbenes - origins, metabolism and potential importance to human health. J. Sci. Food Agric. 80, 1044-1062.

(50) Knight, D. C., and Eden, J. A. (1996) A review of the clinical effects of phytoestrogens. Obstet. Gynecol. 87, 897-904.

(51) Kuiper, G. G. J. M., Lemmen, J. G., Carlsson, B., Corton, J. C., Safe, S. H., van der Saag, P. T., van der Burg, B., and Gustafsson, J.-A. k. (1998) Interaction of Estrogenic Chemicals and Phytoestrogens with Estrogen Receptor $\beta$. Endocrinology 139, 4252-4263.

(52) Markiewicz, L., Garey, J., Adlercreutz, H., and Gurpide, E. (1993) In vitro bioassays of non-steroidal phytoestrogens. J. Steroid Biochem. Mol. Biol. 45, 399-405.

(53) Maglich, J. M., Stoltz, C. M., Goodwin, B., Hawkins-Brown, D., Moore, J. T., and Kliewer, S. A. (2002) Nuclear pregnane X receptor and constitutive androstane receptor regulate overlapping but distinct sets of genes involved in xenobiotic detoxification. Mol. Pharmacol. 62, $638-646$

(54) Nolte, R. T., Wisely, G. B., Westin, S., Cobb, J. E., Lambert, M. H., Kurokawa, R., Rosenfeld, M. G., Willson, T. M., Glass, C. K., and Milburn, M. V. (1998) Ligand binding and co-activator assembly of the peroxisome proliferator-activated receptor-gamma. Nature 395, 137-143.

(55) Hartung, T., and McBride, M. (2011) Food for Thought $\cdots$ on mapping the human toxome. Altex 28, 83-93.

(56) Jassal, B., Matthews, L., Viteri, G., Gong, C., Lorente, P., Fabregat, A., Sidiropoulos, K., Cook, J., Gillespie, M., Haw, R., Loney, F., May, B., Milacic, M., Rothfels, K., Sevilla, C., Shamovsky, V., Shorser, S., Varusai, T., Weiser, J., Wu, G., Stein, L., Hermjakob, H., and D'Eustachio, P. (2019) The reactome pathway knowledgebase. Nucleic Acids Res. 48, D498-D503.

(57) Kanehisa, M., Furumichi, M., Tanabe, M., Sato, Y., and Morishima, K. (2017) KEGG: new perspectives on genomes, pathways, diseases and drugs. Nucleic Acids Res. 45, D353-D361.

(58) Davis, A. P., Grondin, C. J., Johnson, R. J., Sciaky, D., McMorran, R., Wiegers, J., Wiegers, T. C., and Mattingly, C. J. (2019) The Comparative Toxicogenomics Database: update 2019. Nucleic Acids Res. 47, D948-d954.

(59) Miksicek, R. J. (1993) Commonly occurring plant flavonoids have estrogenic activity. Mol. Pharmacol. 44, 37-43.

(60) Silva, M., Pham, N., Lewis, C., Iyer, S., Kwok, E., Solomon, G., and Zeise, L. (2015) A Comparison of ToxCast Test Results with In Vivo and Other In Vitro Endpoints for Neuro, Endocrine, and Developmental Toxicities: A Case Study Using Endosulfan and Methidathion. Birth Defects Res., Part B 104, 71-89.

(61) Reif, D. M., Martin, M. T., Tan, S. W., Houck, K. A., Judson, R. S., Richard, A. M., Knudsen, T. B., Dix, D. J., and Kavlock, R. J. (2010) Endocrine profiling and prioritization of environmental chemicals using ToxCast data. Environ. Health Perspect. 118, 17141720. 
(62) Rotroff, D. M., Dix, D. J., Houck, K. A., Knudsen, T. B., Martin, M. T., McLaurin, K. W., Reif, D. M., Crofton, K. M., Singh, A. V., Xia, M., Huang, R., and Judson, R. S. (2013) Using in vitro high throughput screening assays to identify potential endocrine-disrupting chemicals. Environ. Health Perspect. 121, 7-14.

(63) Judson, R. S., Magpantay, F. M., Chickarmane, V., Haskell, C., Tania, N., Taylor, J., Xia, M., Huang, R., Rotroff, D. M., Filer, D. L., Houck, K. A., Martin, M. T., Sipes, N., Richard, A. M., Mansouri, K., Setzer, R. W., Knudsen, T. B., Crofton, K. M., and Thomas, R. S. (2015) Integrated Model of Chemical Perturbations of a Biological Pathway Using 18 In Vitro High-Throughput Screening Assays for the Estrogen Receptor. Toxicol. Sci. 148, 137-154.

(64) Browne, P., Judson, R. S., Casey, W. M., Kleinstreuer, N. C., and Thomas, R. S. (2015) Screening Chemicals for Estrogen Receptor Bioactivity Using a Computational Model. Environ. Sci. Technol. 49, 8804-8814.

(65) Lecomte, S., Demay, F., Ferrière, F., and Pakdel, F. (2017) Phytochemicals Targeting Estrogen Receptors: Beneficial Rather Than Adverse Effects? Int. J. Mol. Sci. 18, 1381.

(66) Krizova, L., Dadakova, K., Kasparovska, J., and Kasparovsky, T. (2019) Isoflavones. Molecules 24, 1076.

(67) Wetmore, B. A., Wambaugh, J. F., Allen, B., Ferguson, S. S., Sochaski, M. A., Setzer, R. W., Houck, K. A., Strope, C. L., Cantwell, K., Judson, R. S., LeCluyse, E., Clewell, H. J., Thomas, R. S., and Andersen, M. E. (2015) Incorporating High-Throughput Exposure Predictions With Dosimetry-Adjusted In Vitro Bioactivity to Inform Chemical Toxicity Testing. Toxicol. Sci. 148, 121-136.

(68) Escher, B. I., Glauch, L., König, M., Mayer, P., and Schlichting, R. (2019) Baseline Toxicity and Volatility Cutoff in Reporter Gene Assays Used for High-Throughput Screening. Chem. Res. Toxicol. 32, $1646-1655$

(69) Watt, E. D., and Judson, R. S. (2018) Uncertainty quantification in ToxCast high throughput screening. PLoS One 13, 23.

(70) Shah, I., Houck, K., Judson, R. S., Kavlock, R. J., Martin, M. T., Reif, D. M., Wambaugh, J., and Dix, D. J. (2011) Using Nuclear Receptor Activity to Stratify Hepatocarcinogens. PLoS One 6, e14584.

(71) Kleinstreuer, N. C., Judson, R. S., Reif, D. M., Sipes, N. S., Singh, A. V., Chandler, K. J., DeWoskin, R., Dix, D. J., Kavlock, R. J., and Knudsen, T. B. (2011) Environmental Impact on Vascular Development Predicted by High-Throughput Screening. Environ. Health Perspect. 119, 1596-1603.

(72) Janesick, A. S., Dimastrogiovanni, G., Vanek, L., Boulos, C., Chamorro-Garcia, R., Tang, W., and Blumberg, B. (2016) On the Utility of ToxCast and ToxPi as Methods for Identifying New Obesogens. Environ. Health Perspect. 124, 1214-1226.

(73) Thomas, R. S., Black, M. B., Li, L., Healy, E., Chu, T.-M., Bao, W., Andersen, M. E., and Wolfinger, R. D. (2012) A Comprehensive Statistical Analysis of Predicting In Vivo Hazard Using HighThroughput In Vitro Screening. Toxicol. Sci. 128, 398-417. 